\title{
Postnatal Development of the Rat Dorsal Funiculus
}

\author{
Kyungsoon Chung and Richard E. Coggeshall \\ Marine Biomedical Institute and Departments of Physiology and Biophysics, and Anatomy and Neurosciences, University \\ of Texas Medical Branch, Galveston, Texas 77550
}

\begin{abstract}
The present study shows that there are approximately 21,000 axons in the neonatal rat dorsal funiculus, as compared to 26,000 in 2-week-old animals. We attribute this increase primarily to arriving corticospinal fibers. In adult animals, however, there are approximately 15,000 axons. This is a decline of $58 \%$ from the 2 week level, and the decrease is proportionately similar in the corticospinal area and the dorsal funiculus proper. Thus, axon numbers decline in later postnatal development, and since the decline seems to be well past the time of the histogenetic death of the cells that give rise to these axons, we propose that the loss is not caused by the death of neurons. This is further evidence that a reduction in axon numbers not accompanied by cell death is a widespread phenomenon in mammalian postnatal neural development. We infer that the mechanism of axon loss is a reduction in axon branching and that its function is to sharpen synaptic connections.
\end{abstract}

The death of a significant number of newly formed neurons in early development is an important regressive process in the formation of the nervous system. Another regressive process, presumably of equal importance, would be a loss of axons not caused by this neuronal death. There is some evidence that this occurs (see Discussion), but the generality of the phenomenon has not been established. In particular, the large funiculi that make up the white matter in the mammalian spinal cord have not been studied from this point of view. The dorsal funiculus of the rat spinal cord is of particular interest because of the organization of primary afferent fibers in this area; also, this pathway offers a favorable opportunity for assaying sprouting. Thus it would be desirable to determine the number of axons in the normal rat dorsal funiculus at various times during postnatal development, both to evaluate an important developmental process and as a basis for further studies on the organization and modification of an important spinal pathway.

\section{Materials and Methods}

Pregnant albino rats were obtained from Texas Inbred Mice Co., Houston, TX. The rats were killed 1 day (neonates) and 2 weeks after birth. Adult rats (between 2 and 9 months after birth) were also killed. Methods of fixation and examination of the tissue have been published previously (Chung and Coggeshall, 1984). In brief, newborn animals were

\footnotetext{
Received Mar. 13, 1986; revised Oct. 18, 1986; accepted Oct. 19, 1986.

This work is supported by NIH Grants 17039,11061 , and 11255 ; by the Muscular Dystrophy Association; and by a grant from the Texas Neurofibromatosis Foundation.

Correspondence should be addressed to Kyungsoon Chung, Ph.D., Marine Biomedical Institute, 200 University Boulevard \#532, Galveston, TX 775502772.

Copyright (C 1987 Society for Neuroscience $0270-6474 / 87 / 040972-06 \$ 02.00 / 0$
}

anesthetized by hypothermia (Hulsebosch and Coggeshall, 1983) and the others with sodium pentobarbital (Nembutal), $35 \mathrm{mg} / \mathrm{kg}$. When anesthesia was deep, $0.9 \% \mathrm{NaCl}$, with 200 units of heparin and $0.5 \%$ $\mathrm{NaNO}_{2}$ per $100 \mathrm{ml}$, was perfused until the right auricular effluent was free of blood. Then the fixative was changed to $3 \%$ glutaraldehyde, $3 \%$ paraformaldehyde, and $0.1 \%$ picric acid in cacodylate buffer, $\mathrm{pH} 7.4$ for 2 week and older rats, and $2 \%$ glutaraldehyde, $2 \%$ paraformaldehyde, and $0.1 \%$ picric acid in cacodylate buffer, $\mathrm{pH} 7.4$, for the neonates. After fixation, the spinal cord was removed, the $\mathbf{S} 2$ segment was blocked and placed in $1 \%$ osmium tetroxide and $1.5 \%$ potassium ferricyanide (Langford and Coggeshall, 1980), rinsed, dehydrated, embedded, sectioned with glass or diamond knives, and photographed in a Philips 300 electron microscope. Montages were made and all axons in the dorsal column, in both corticospinal and noncorticospinal areas, were counted.

\section{Results}

The dorsal funiculus is the spinal white matter between the dorsal horns (Fig. 1). It extends from the dorsal midline to the medial edge of Lissauer's tract (dorsolateral fasciculus) (Fig. 1). In the rat, the large majority of corticospinal fibers descend in a compact bundle in the ventromedial part of each dorsal funiculus. The myelinated fibers in corticospinal areas are considerably smaller than those in the rest of the dorsal funiculus. This allows the tract to be recognized in adult (Fig. 1A) and juvenile (Fig. $1 B$ ) animals. The rest of the dorsal funiculus, which is that part outside the corticospinal tract, will be referred to as the "dorsal funiculus proper." In the newborn animal, there are no myelinated axons, so the distinction between the dorsal funiculus proper and the corticospinal tract cannot be made. The neural components of these pathways are myelinated and unmyelinated axons, which have been illustrated several times by various investigators (e.g., Chung and Coggeshall, 1985). In the neonate, the dorsal funiculus consists of closely apposed, relatively circular axons separated into large bundles by radial astrocytic processes (Fig. 2) (Schreyer and Jones, 1982).

Counts from the dorsal funiculi of the second segment of sacral spinal cord ( $\mathrm{S} 2)$ of rats at various ages are given in Table 1. Note that there are approximately $21,000(20,813 \pm 1222)$ axons in the dorsal funiculus in the neonate, and that in these animals no distinction can be made between the corticospinal tract and the dorsal funiculus proper. In 2 week animals, the total number of axons has risen to approximately 26,000 $(26,053 \pm 3391)$, with approximately one-third in the corticospinal area and the others in the dorsal funiculus proper. In the adult, the total numbers have declined to approximately $15,000(15,158 \pm 336)$, with a slightly greater, but not significantly different, relative decline in the corticospinal area compared to that in the dorsal funiculus proper. These data emphasize the substantial contribution of unmyelinated fibers to the adult dorsal funiculi. 

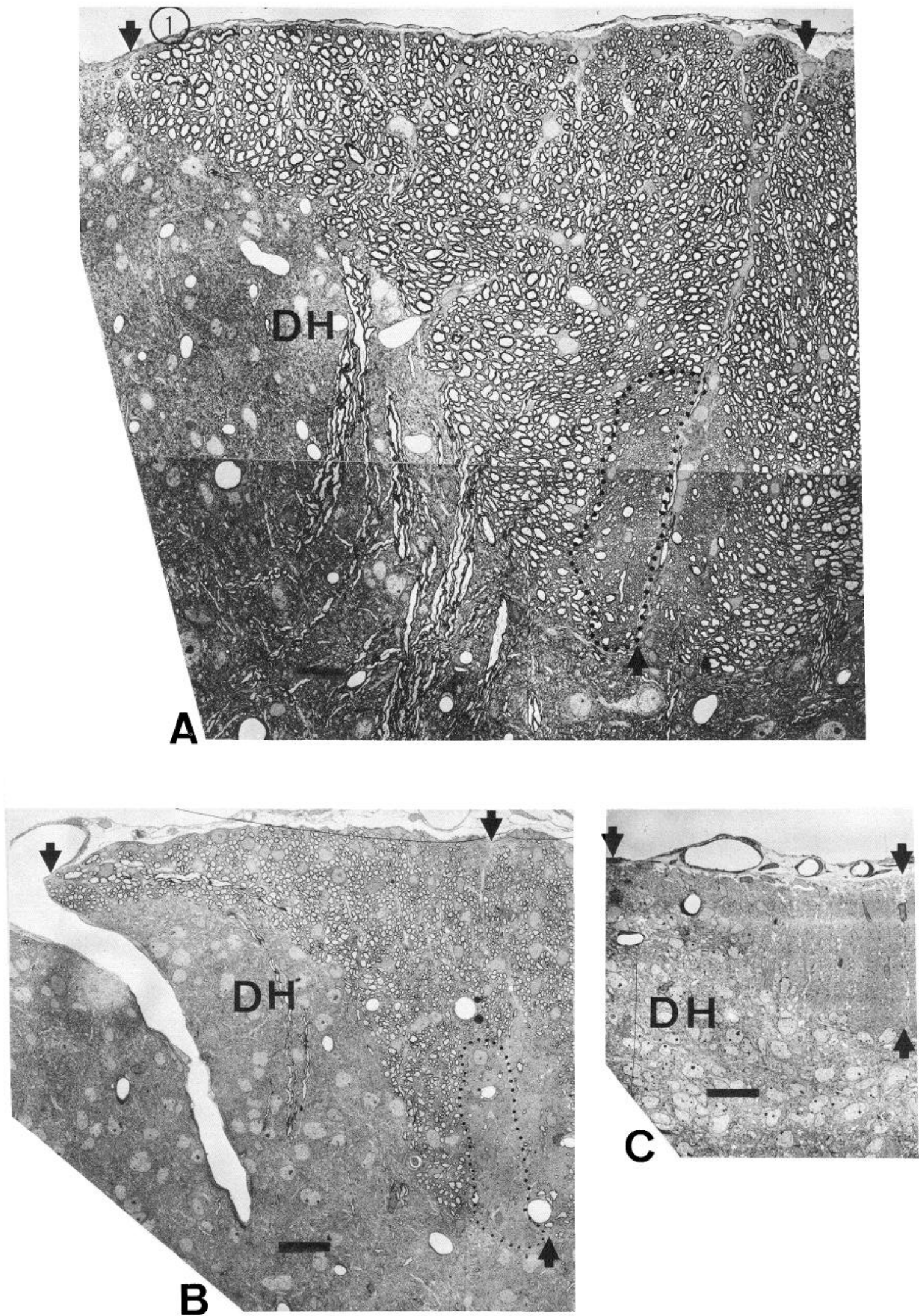

Figure 1. Low-power electron micrographs of the dorsal funiculus of adult $(A)$, 2-week-old $(B)$, and neonatal $(C)$ rats. Arrows indicate boundaries of the dorsal funiculus; the dorsal boundary is the pia mater; the medial boundary is the dorsal median septum (indicated by 2 vertically arranged arrows on the right side of each picture); the lateral and ventral boundaries are Lissauer's tract (arrow, the upper left corner) and the dorsal horn $(D H)$. In the adult and 2 week rats the corticospinal tract is outlined. Bar, $20 \mu \mathrm{m}$. 
Figure 2. Electron micrograph of unmyelinated axons (asterisks) and astrocytic processes (arrows) in newborn rat dorsal funiculus. Bar, $1 \mu \mathrm{m}$.

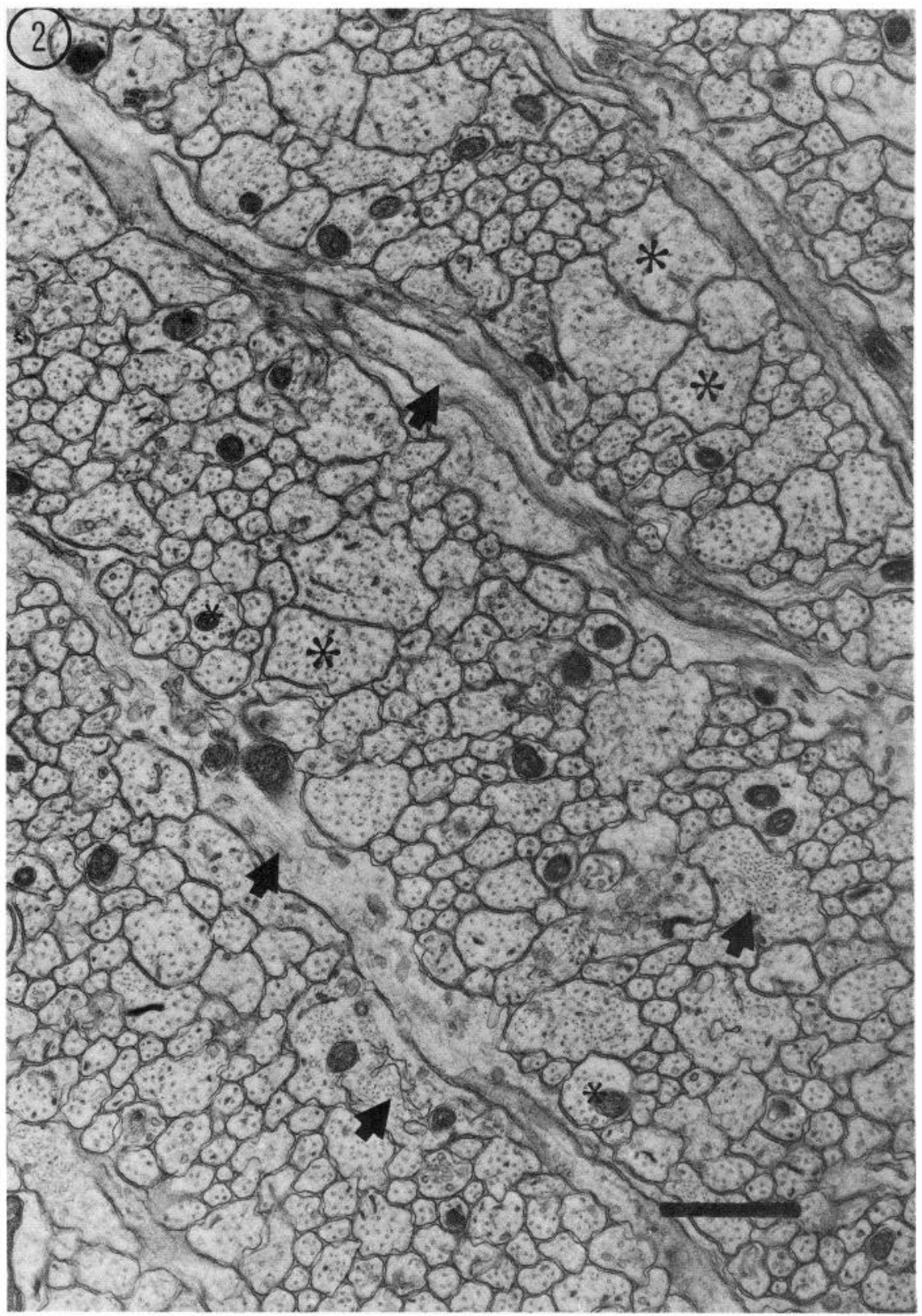

\section{Discussion}

Neural development is usually described in terms of progressive events, such as cell proliferation and synapse formation, but regressive events are now thought to be equally, or almost equally, important (e.g., Jacobson, 1978; Cowan et al., 1984; Purves and Lichtman, 1985). The most examined regressive event in mammals is the histogenetic cell death that occurs prenatally in almost all areas studied to date (e.g., Cowan et al., 1984). Axon numbers were not considered in the above studies, but remodeling events, such as a loss of axons, would undoubtedly have been interpreted as a consequence of the cell loss. That axon loss could occur without cell loss was probably first suggested in physiologic studies that indicated a postnatal loss (with a consequent sharpening of remaining connections) of inputs for populations as diverse as somatic muscle cells, cells in peripheral visceral ganglia, and Purkinje cells of the cerebellum (summarized in Cowan et al., 1984). Since the sharpening of connections occurs postnatally, it has not been considered a result of the loss of neurons, but rather of the local withdrawal of presynaptic processes ("fine tuning"; Cowan et al., 1984), or of the loss of axons ("long axon collaterals"; Cowan et al., 1984).

That axon loss not caused by cell death occurs in normal postnatal development has recently been suggested for cortical systems. The findings are that many cells, widely distributed throughout the cerebral cortex, are labeled by retrogradely transported markers applied to the newborn, but that seemingly fewer cells, in more restricted locations, are labeled when the marker is applied to the adult (Innocenti, 1981; O'Leary et al., 1981; Ivy and Killackey, 1982). If newborns are labeled as above but not killed before adulthood, the labeled cells are still widely distributed and seemingly as numerous as after animals are killed early (O'Leary et al., 1981; Cowan et al., 1984). The labeling of fewer cells in more restricted locations when marker 
Table 1. Numbers of myelinated (M) and unmyelinated (U) axons in the corticospinal tract and the rest of the dorsal funiculus (dorsal funiculus proper) in neonatal, 2-week-old, and adult rat"

\begin{tabular}{|c|c|c|c|c|c|c|c|}
\hline \multirow[b]{2}{*}{ Animal } & \multicolumn{3}{|c|}{ Dorsal funiculus proper } & \multicolumn{3}{|c|}{ Corticospinal tract } & \multirow[b]{2}{*}{ Total } \\
\hline & $\bar{M}$ & $\mathrm{U}$ & Total & $\mathbf{M}$ & $\mathbf{U}$ & Total & \\
\hline Neonate (1) & - & 22,184 & 22,184 & - & - & - & 22,184 \\
\hline Neonate (2) & - & 20,414 & 20,414 & - & - & - & 20,414 \\
\hline Neonate (3) & - & 19,840 & 19,840 & - & - & - & 19,840 \\
\hline Mean \pm SD & & & $20,813 \pm 1222$ & & & & $20,813 \pm 1222$ \\
\hline Week 2 (1) & 2087 & 17,334 & 19,421 & 102 & 8552 & 8654 & 28,075 \\
\hline Week 2 (2) & 2468 & 12,327 & 14,795 & 134 & 6638 & 6772 & 21,567 \\
\hline Week $2(3)$ & 2263 & 16,045 & 18,308 & 72 & 10,809 & 10,881 & 29,189 \\
\hline Week 2 (4) & 2645 & 13,832 & 16,477 & 114 & 8790 & 8904 & 25,381 \\
\hline Mean \pm SD & & & $17,250 \pm 2038$ & & & $8803 \pm 1681$ & $26,053 \pm 3391$ \\
\hline Adult (1) & 6580 & 3237 & 9817 & 2241 & 2706 & 4947 & 14,764 \\
\hline Adult (2) & 6700 & 3787 & 10,487 & 1595 & 2912 & 4507 & 14,994 \\
\hline Adult (3) & 6796 & 4004 & 10,800 & 1886 & 2774 & 4660 & 15,460 \\
\hline Adult (4) & 7000 & 3515 & 10,515 & 2272 & 2626 & 4898 & 15,413 \\
\hline Mean $\pm \mathrm{SD}$ & & & $10,405 \pm 417$ & & & $4753 \pm 206$ & $15,158 \pm 336$ \\
\hline
\end{tabular}

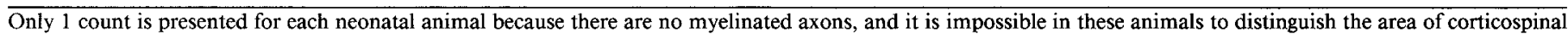
tract from the rest of the dorsal funiculus.

is applied to older animals is interpreted as implying that some cells no longer send axons to the area where the marker was placed, and the seeming persistence into adulthood of all cells labeled at birth is interpreted as indicating that there is no loss of labeled cells. The conclusion follows that there is axon loss or at least axon withdrawal, but that this loss is not associated with the loss of cells. Although this reasoning is extremely suggestive, there are problems. For example, there may be differences in the transport of markers between neonatal and adult axons (Ivy et al., 1984); postnatal death of cells in the cerebral cortex has been reported (Pearlman, 1984); and the lack of counts renders conclusions based on the number of labeled cells only preliminary impressions.

Another way to determine whether axons are lost during development is to count them at different postnatal times. Aguayo et al. (1973), investigating descriptions of the spontaneous loss of axons in normal peripheral nerves (Webster and O'Connell, 1970; Reier and Hughes, 1972), noted 16,000 axons in the cervical sympathetic trunk at birth and only 5760 axons 3 weeks later. A decline of similar magnitude was reported by Koppel and Innocenti (1983): 70\% of callosal axons present at birth were eliminated by adulthood. Similarly, there is a significant reduction in the number of corticospinal axons after birth (Reh and Kalil, 1982) and of optic axons in neonates and after birth (Ng and Stone, 1982; Rakic and Riley, 1983; Williams et al., 1983, 1986). It is unlikely that losses of this magnitude could be explained solely on the basis of cell death, especially since few neurons are supposed to be lost after birth; however, there has been debate as to whether some sympathetic cells are lost postnatally (Hendry and Campbell, 1976; Davies, 1978; Smolen et al., 1983) and there are reports of cell death in the cerebral cortex after birth (Pearlman, 1984).

In our own work, we have shown a significant postnatal decrease in the number of axons in dorsal roots, sciatic nerve, and dorsolateral fasciculus (Lissauer's tract), and these decreases have taken place much later than the normal prenatal death among neuronal populations that give rise to these axons (Chung and Coggeshall, 1984; Hulsebosch et al., 1986; Jenq et al., 1986). A difficulty with Lissauer's tract, however, is that its ventral border is difficult to recognize in newborn animals; thus one of the reasons for the present study is to determine changes in axon numbers in one of the large funiculi of the spinal white matter, where the boundaries are clear even in the newborn.

The present study is concerned with the dorsal funiculus of the rat. The rat dorsal funiculus, like that of other mammals, contains coarse primary afferent fibers, second-order afferent fibers, and propriospinal fibers. In addition, in the rat, most of the corticospinal axons travel caudally in the ventral part of the dorsal funiculus, and in the adult they can be recognized in cross sections as a distinctive oval area of fine myelinated and unmyelinated axons, surrounded and emphasized by the larger myelinated axons of the primary afferent system (Schreyer and Jones, 1982). For this reason we are able to present 2 counts for older animals, one for the corticospinal area and the other for the rest of the tract (dorsal funiculus proper). Unfortunately, these distinctions blur in younger animals, where fibers are small and relatively few, or none, are myelinated; thus a separate count for the corticospinal system can be given only for older animals. Finally, there is a significant population of unmyelinated primary afferent fibers in cat dorsal funiculus (Chung and Coggeshall, 1985), and the same is true in rats.

Before proceeding, our counts should be discussed. A problem in larger systems is that not all axons can be counted, so that sampling must be done; also, boundaries are not always clear. In rat dorsal columns, however, all axons are counted, which eliminates sampling, and the boundaries are clear. The only other possible difficulty is in recognizing the axons and distinguishing them from supporting cell processes. However, myelinated axons are not a problem, since they have a characteristic sheath, and unmyelinated axons have clear cytologic characteristics (e.g., Peters et al., 1976; Chung and Coggeshall, 1982). Furthermore, the astrocytes, whose processes are most likely to be confused with axons, contain more filaments per unit of cytoplasm than do axons, although this distinction is clearer in older animals. The glial processes are still generally larger than axons in neonates; they have an irregular shape, and are usually 
radially oriented, in contrast to the smaller, round, longitudinally oriented axons. Thus, even in newborns, we feel the counts are reasonably accurate.

In the present study, the results do not indicate a simple decline in axon numbers from birth to adulthood in the rat dorsal funiculus. Rather there is an increase from birth to 2 weeks of age, and then a relatively striking decrease from 2 weeks to adulthood. These 2 intervals will be discussed separately.

\section{Postnatal days 0-14}

The period from postnatal day 0 to 14 is characterized by an increase in axonal numbers in the dorsal funiculus. We assume that the primary afferent and propriospinal fibers are present in the dorsal funiculus at birth, but that corticospinal axons do not reach sacral levels of the spinal cord until approximately day 9 after birth (Schreyer and Jones, 1982). If we drew an analogy between the propriospinal and primary afferent fibers in the dorsal funiculus and similar fibers in Lissauer's tract (Chung and Coggeshall, 1984), we would assume that there was a relatively steady reduction in these fibers after birth. For the first 2 weeks after birth, however, we assume that the influx of corticospinal fibers keeps the total number of fibers increasing. Thus, this is not a static system and an expected decrease in primary afferent and propriospinal axons is more than balanced by an influx of corticospinal axons.

\section{Day 14 to adulthood}

The dorsal funiculi in animals $14 \mathrm{~d}$ old and older are distinguishable from those of younger animals by their recognizable corticospinal area and the considerable decrease in axon numbers in both corticospinal and noncorticospinal areas of the dorsal funiculus as the animals age. The decrease in axonal numbers is proportionately similar in the 2 areas, and the total decrease is from approximately 26,000 at 2 weeks of age to 15,000 in adulthood. Since this is a sizable loss and it occurs throughout the rat dorsal funiculus, we assume that all known axon types in the path-primary afferents, second-order afferents, propriospinal and corticospinal fibers - are diminishing in number during this time period. Thus our primary question as to whether fibers in the rat dorsal funiculus show a significant postnatal decline has been answered, with the further observation that the decline is only manifest after 2 weeks of age.

\section{Is process loss associated with cell loss?}

The cells that give rise to the fibers in the dorsal funiculus are primary afferent neurons, second-order afferent neurons, propriospinal neurons, and corticospinal neurons. There is no evidence for the death of significant numbers of primary afferent (Hulsebosch et al., 1986) or intraspinal neurons at the time axon numbers are declining. Mammalian primary afferent neurons, for example, proliferate and then presumably lose their excess numbers well before birth (Lawson et al., 1974; Forbes and Welt, 1981). Furthermore, although postnatal counts of these neurons are not in complete agreement, any debate concerns whether they are constant in number or increase during postnatal lifenot whether they decrease (Hatai, 1902; Cavanaugh, 1951; Emery and Singhal, 1973; Ohta et al., 1974; Lawson, 1979; Devor and Gorvrin-Lippmann, 1985; Hulsebosch et al., 1986). It is difficult to obtain accurate total counts of intraspinal or corticospinal cells because of their overwhelming numbers and the difficulty in establishing exact boundaries, but to our knowledge there are no reports of significant loss of intraspinal neurons in normal neonatal mammals. The death of cortical cells in neonatal mammals has been reported (Pearlman, 1984), but the numbers are sinall compared to those of corticospinal axon reduction, and the process is over before 2 weeks of postnatal age in mice (Pearlman, 1984). Furthermore, corticospinal neurons are not reported to decrease numerically if labeled at birth (e.g., Cowan et al., 1984). Accordingly, it seems safe to assume that the loss of processes in the dorsal funiculus is not caused by the death of the cells that give rise to these processes. Thus, we conclude that axon reduction in the dorsal column is not due to the death of the neurons that generated these axons.

If we accept the above reasoning, this study provides further evidence for the suggestion that axon loss not associated with cell death may be a general process in mammalian neural development. Axon loss has been shown, for example, in several fiber systems associated with the cerebral cortex, in the optic and sciatic nerves, in the dorsal roots, in the sympathetic trunk, in the pyramidal tract, in Lissauer's tract, and now in the dorsal funiculus of the spinal cord. In these areas postnatal axon loss is sizable and, if previous arguments are accepted, not associated with cell loss. Then the only obvious possible explanation for this diminution in axon numbers is that it is the result of reduced axonal branching. A reduction in branching would presumably lessen multiple inputs and sharpen connections, not by local remodeling but by elimination of large numbers of axons at a considerable distance from their target. A possibly useful consequence would be that this process of axon loss could be modified by experience (Innocenti et al., 1985). There is also the considerable increase in dorsal root axon numbers in neonates, as compared to adults, after spinal denervation or removal of nerve growth factor (Hulsebosch et al., 1984). The interpretation of the latter phenomenon has been that sprouting occurred, but it now seems equally possible that normal axon loss was prevented. Further exploration of the phenomenon of neonatal axon loss is thus warranted, and since a primary goal of developing neurons is to establish correct connections, a process whereby axons are removed is probably as, or more, important than a process whereby cells are removed.

\section{References}

Aguayo, A. J., L. C. Terry, and G. M. Bray (1973) Spontaneous loss of axons in sympathetic unmyelinated nerve fibers of the rat during development. Brain Res. 54: 360-364.

Cavanaugh, M. W. (1951) Quantitative effects of peripheral innervation area on nerves and spinal ganglion cells. J. Comp. Neurol. 94: $181-219$.

Chung, K., and R. E. Coggeshall (1982) Quantitation of propriospinal fibers in the tract of Lissauer of the rat. J. Comp. Neurol. 211: 418426.

Chung, K., and R. E. Coggeshall (1984) The postnatal development of the tract of Lissauer in the rat. J. Comp. Neurol. 229: 471-475.

Chung, K., and R. E. Coggeshall (1985) Unmyelinated primary afferent fibers in dorsal funiculi of cat sacral spinal cord. J. Comp. Neurol. 238: 365-369.

Cowan, W. M., J. W. Fawcett, D. D. M. O'Leary, and B. B. Stanfield (1984) Regressive events in neurogenesis. Science 225: 1258-1265.

Davies, D. C. (1978) Neuronal numbers in the superior cervical ganglion of the neonatal rat. J. Anat. 127: 43-51.

Devor, M., and R. G. Gorvrin-Lippmann (1985) Neurogenesis in adult rat dorsal root ganglia. Neurosci. Lett. 61: 189-194.

Emery, J. L., and R. Singhal (1973) Changes associated with growth in the cells of the dorsal root ganglion in children. Dev. Med. Child Neurol 15: 460-467. 
Forbes, D. J., and C. Welt (1981) Neurogenesis in the trigeminal ganglion of the albino rat. A quantitative autoradiography study. $\mathrm{J}$. Comp. Neurol. 199: 133-147.

Hatai, S. (1902) Number and size of the spinal ganglion cells and dorsal root fibers in the white rat at different ages. J. Comp. Neurol. 12: 107-124.

Hendry, I. A., and J. Campbell (1976) Morphometric analysis of rat superior cervical ganglion after axotomy and nerve growth factor treatment. J. Neurocytol. 5: 351-360.

Hulsebosch, C. E., and R. E. Coggeshall (1983) A comparison of axonal numbers in dorsal roots following spinal cord hemisection in neonate and adult rats. Brain Res. 263: 187-197.

Hulsebosch, C. E., R. E. Coggeshall, and J. R. Perez-Polo (1984) Increased numbers of thoracic dorsal root axons in rats given antibodies to nerve growth factor. Science 225: 525-526.

Hulsebosch, C. E., R. E. Coggeshall, and K. Chung (1986) Numbers of rat dorsal root axons and ganglion cells during postnatal development. Dev. Brain Res. 2: 105-113.

Innocenti, G. M. (1981) Growth and reshaping of axons in the establishment of visual callosal connections. Science 212: 825-827.

Innocenti, G. M., D. O. Frost, and J. Illes (1985) Maturation of visual callosal connections in visually deprived kittens: A challenging critical period. J. Neurosci. 5: 265-267.

Ivy, G. O., and H. P. Killackey (1982) Ontogenetic changes in the projections of neocortical neurons. J. Neurosci. 2: 735-743.

Ivy, G. O., H. J. Gould, III, and H. P. Killackey (1984) Variability in the distribution of callosal projection neurons in the adult rat parietal cortex. Brain Res. 306: 53-61.

Jacobson, M. (1978) Developmental Neurobiology, pp. 253-308, Plenum, New York and London.

Jenq, C.-B., K. Chung, and R. E. Coggeshall (1986) Postnatal loss of axons in normal rat sciatic nerve. J. Comp. Neurol. 244: 445-450.

Koppel, H., and G. M. Innocenti (1983) Is there a genuine exuberancy of callosal projections in development? A quantitative electron microscopic study in the cat. Neurosci. Lett. 41: 33-40.

Langford, L. A., and R. E. Coggeshall (1980) The use of potassium ferricyanide in neural fixation. Anat. Rec. 197: 297-303.

Lawson, S. H. (1979) The postnatal development of large light and small dark neurons in mouse dorsal root ganglia: A statistical analysis of cell number and size. J. Neurocytol. 8: 275-294.

Lawson, S. H., K. W. Caddy, and T. J. Biscoe (1974) Development of rat dorsal root ganglion neurones. Studies of cell birthdays and changes in mean cell diameter. Cell Tissue Res. 153: 399-413.
Ng, A. Y. K., and J. Stone (1982) The optic nerve of the cat: Appearance and loss of axons during normal development. Dev. Brain Res. 5: 263-271.

Ohta, M., K. Offord, and P. J. Dyck (1974) Morphometric evaluation of the first sacral ganglia of man. J. Neurol. Sci. 22: 73-82.

O'Leary, D. D. M., B. B. Stanfield, and W. M. Cowan (1981) Evidence that the early postnatal restriction of the cells of origin of the collosal projection is due to the elimination of axonal collaterals rather than to the death of neurons. Dev. Brain Res. 1: 607-617.

Pearlman, A. L. (1984) The visual cortex of the normal mouse and the reeler mutant. In Cerebral Cortex, vol. 3, A. Peters and E. G. Jones, eds., pp. 1-18, Plenum, New York and London.

Peters, A., S. Palay, and H. deF. Webster (1976) The Fine Structure of the Nervous System: The Neurons and Supporting Cells, Saunders, Philadelphia, London, Toronto.

Purves, D., and J. W. Lichtman (1985) Principles of Neural Development, Sinauer, Sunderland, MA.

Rakic, P., and K. P. Riley (1983) Overproduction and elimination of retinal axons in the fetal rhesus monkey. Science 219: 1441-1444.

Reh, T., and K. Kalil (1982) Development of the pyramidal tract in the hamster. II. An electron microscopic study. J. Comp. Neurol. 205: 77-88.

Reier, P. J., and A. Hughes (1972) Evidence for spontaneous axon degeneration during peripheral nerve maturation. Am. J. Anat. 135: 147-152.

Schreyer, D. J., and E. G. Jones (1982) Growth and target finding by axons of the corticospinal tract in prenatal and postnatal rats. Neuroscience 7: 1837-1853.

Smolen, A. J., L. L. Wright, and T. J. Cunningham (1983) Neuron numbers in the superior cervical sympathetic ganglion of the rat: A critical comparison of methods for cell counting. J. Neurocytol. 12: 739-750.

Webster, H. deF., and M. F. O'Connell (1970) Myelin formation in peripheral nerves. A morphological reappraisal and its neuropathological significance. In Proceedings of the VIth International Congress of Neuropathology, pp. 579-588, Masson, Paris.

Williams, R. W., M. J. Bastiani, and L. M. Chalupa (1983) Loss of axons in the cat optic nerve following fetal unilateral enucleation: An electron microscopic analysis. J. Neurosci. 3: 133-144.

Williams, R. W., M. J. Bastiani, B. Lia, and L. M. Chalupa (1986) Growth cones, dying axons, and development fluctuations in the fiber population of the cat's optic nerve. J. Comp. Neurol. 246: 32-69. 